\title{
The Second Gelfand-Dickey Bracket as a Bracket on a Poisson-Lie Grassmannian
}

\author{
Ilya Zakharevich \\ Department of Mathematics, M.I.T., Cambridge, MA 02139, USA \\ Received: December 1, 1992
}

Dedicated to Israel M. Gelfand at his $80^{\text {th }}$ birthday

\begin{abstract}
We introduce a Poisson structure on a Grassmannian $\operatorname{Gr}_{k}(V)$ on which the Poisson-Lie group GL $(V)$ acts in a Poisson-Lie way. We discuss the analytic complications connected with the infinite-dimensional case $V=C^{\infty}(\mathbb{R})$ and show that an open subset of $\mathrm{Gr}_{k}(V)$ with this Poisson structure is isomorphic to the Gelfand-Dickey manifold of differential operators of order $k$ with the second Gelfand-Dickey bracket. In fact we introduce as a consequence a Poisson-Lie action of an enormous group on the Gelfand-Dickey manifold generalizing (on the semiclassical level) the Sugavara inclusion.
\end{abstract}

\section{Contents}

0. Introduction . . . . . . . . . . . . . . . . . . . . . . . . . . . 94

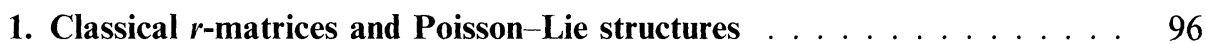

1.1. The classical Yang-Baxter equation . . . . . . . . . . . . 96

1.2. Poisson manifolds . . . . . . . . . . . . . . . . . . . 97

1.3. Skewsymmetric $r$-matrices . . . . . . . . . . . . . . . 98

1.4. The Modified Yang-Baxter equation . . . . . . . . . . . . . . 100

1.5. Homogeneous spaces with Poisson-Lie action . . . . . . . . . 102

2. Gelfand-Dickey brackets . . . . . . . . . . . . . . . . . . . . . . . 104

2.0. The second Gelfand-Dickey bracket on the set of differential

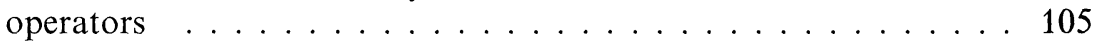

2.1. The identification with a Grassmannian . . . . . . . . . . . 107

2.2. A Poisson-Lie algebra of differential operators as a Poisson-Lie subalgebra of $g l \ldots \ldots \ldots \ldots 111$

2.3. The periodical case . . . . . . . . . . . . . 112

2.4. The matrix case . . . . . . . . . . . . . . . . . . 114

2.5. A conjecture on quantization: the Kac-Moody case . . . . . . . . 115

2.6. The topological approach . . . . . . . . . . . . . . 116

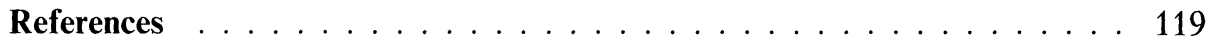




\section{Introduction}

In this paper we consider the manifold of differential operators of order $n$ with the leading term $\partial^{n}$. There is a remarkable Poisson structure on this manifold, introduced by Adler [1] (the proof that this structure satisfies the Jacobi relation is due to Gelfand and Dickey [5]). This Poisson bracket on this manifold is usually called the second Gelfand-Dickey structure. ${ }^{1}$ The usual geometrical interpretation comes from identification of this Poisson manifold with the Hamiltonian reduction from the dual space of an affine Lie coalgebra [3].

A new topic in the theory of this Poisson manifold was introduced by Radul, who invented an action of the Lie algebra of differential operators on this manifold. This action does not preserve the Poisson structure on this manifold, however, it changes it in a quite regular way. This kind of action was introduced first in the theory of quantum groups (on the semiclassical level) and is called a Poisson-Lie action. For this notion to be correct, we should first determine a structure of bialgebra on this Lie algebra, or, what is the same, a compatible structure of Lie algebra on the dual space to this Lie algebra.

However, the dual space to the Lie algebra of differential operators is the space of pseudodifferential operators and this space carries a natural structure of Lie algebra. In fact this structure is compatible with the Lie bracket on the algebra. That means that we can correctly define a notion of a Poisson-Lie action of this algebra on a Poisson manifold.

It was a great surprise when (inspired by A. Givental) Radul discovered that the specified above action is indeed a Poisson-Lie action. It seems that it is not easy to explain this fact using the Poisson reduction from the dual space to affine coalgebra.

In this paper we introduce another geometrical interpretation of the second Gelfand-Dickey structure. We introduce an enormous group $\mathrm{GL}\left(C^{\infty}(\mathbb{R})\right)$, consider the usual GL-type Poisson-Lie structure on this group and consider a homogeneous space for this group, the Grassmannian of $n$-dimensional subspaces in $C^{\infty}(\mathbb{R})$. The Poisson structure on the group induces some Poisson structure on this quotient by a parabolic subgroup.

After that it is a good idea to try to write down this Poisson structure in some coordinate system on this Grassmannian. We use the following coordinate system: we identify a differential operator of order $n$ with leading term $\partial^{n}$ with the $(n$ dimensional) space of its solutions in $C^{\infty}(\mathbb{R})$. It is easy to see that this correspondence is $1-1$ on an open subset of a Grassmannian (some Wronskian should be non-zero everywhere for a given $n$-dimensional subspace to be a space of solutions of a differential equation). We compute the Poisson structure in this coordinate system (indeed, the space of differential operators with the leading term $\partial^{n}$ is an affine space, so we can consider this identification as a coordinate system). What we get is the second Gelfand-Dickey structure!

Therefore we got the following fact: there is a Poisson-Lie action of an enormous group $\operatorname{GL}\left(C^{\infty}(\mathbb{R})\right)$ on the second Gelfand-Dickey structure! ${ }^{2}$ Now to explain the

\footnotetext{
1 The first Gelfand-Dickey structure is another topic

2 As it often happens with actions of Poisson-Lie groups, it is a local action: the action of "big" elements of the group can force a point "to get out of the manifold it lives on." Say, we identify the Gelfand-Dickey manifold with an open subset of the Grassmannian $\operatorname{Gr}\left(C^{\infty}(\mathbb{R})\right)$, but this open subset is not stable with respect to the action of $\operatorname{GL}\left(C^{\infty}(\mathbb{R})\right)$
} 
Radul action we should only note that the Lie algebra of differential operators is in fact a Poisson-Lie subalgebra of the Lie bialgebra $\mathrm{gl}\left(C^{\infty}(\mathbb{R})\right)$. Therefore the restriction of the action on this subalgebra is (essentially by definition!) also a Poisson-Lie action.

The last fact is in fact not very obvious. At first sight the bialgebra structure on the space of differential operators has nothing to do with the decomposition of an operator into a sum of a raising and a lowering Volterra operator, that is used in the definition of the Poisson-Lie structure on $\operatorname{GL}\left(C^{\infty}(\mathbb{R})\right)$. However, the former structure is indeed a restriction of the latter, and the proof of this fact is given in Sect. 2.2.

In fact what is announced here is just a simple calculation. The main inspiration for this calculation was the Radul action. To go from the Radul result to the results discussed here, we should only note that the Radul action can be extended from the Lie algebra of differential operators to the Lie algebra of all operators in $C^{\infty}(\mathbb{R})$ if we introduce the identification of the Grassmannian with the set of differential operators. After that we noted that this action is evidently (if the calculation in the Sect. 2.1 can be called a calculation in two rows, and it is, if we drop all the preliminary definitions) a Poisson-Lie action, and the Lie subalgebra of differential operators is a Poisson-Lie subalgebra.

Another point of view on the identification of the Grassmannian with the set of differential operators is given in the paper [6]. It is shown there that this mapping is just a nonlinear momentum mapping for the action of the bialgebra of differential operators. Let us recall that the usual momentum mapping is defined in the case when the action of the Lie algebra preserves the symplectic structure. It sends a manifold into the dual space of some central extension of the Lie algebra. In the case of the Poisson-Lie action such construction is possible at least in some cases; however, this mapping sends the manifold to the dual Lie group to some central extension. We do not want to discuss this notion now, and want only to note that in the case of Lie algebra of differential operators this dual group is an extension of the group corresponding to the Lie algebra of integral operators by one-parametric group $\partial^{s}=e^{s \cdot \log \partial}, s \in \mathbb{R}$. Therefore an element of this group looks like

$$
\left(1+u_{1} \partial^{-1}+u_{2} \partial^{-2}+\cdots\right) \partial^{s} .
$$

The main result of the paper [6] is that the momentum mapping sends an element of the Grassmannian into the corresponding differential operator, i.e., $s=k$ and $u_{l}=0$ for $l>k$. Therefore the above identification is just a momentum mapping.

This construction explains in particular why we can choose a coordinate system on the Grassmannian $\mathrm{Gr}_{k}\left(C^{\infty}(\mathbb{R})\right)$ in such a way that the Poisson bracket becomes quadratic. Indeed, such a coordinate system is given by the nonlinear momentum mapping, at least if it is "almost surjective."

I am indebted to a lot of people for fruitful discussions and inestimable help, among them I.M. Gelfand, A. Givental, A. Goncharov, D. Kazhdan, B. Khesin, M. Kontsevich, O. Kravchenko, J.-H. Lu, H. McKean, A. Radul, N. Reshetikhin. This article couldn't appear without the hospitality of the Harvard University, the MSRI and the MIT during the (extended) period of preparation of this paper.

The structure of this paper is the following: in Sect. 1 we discuss the usual notions from the theory of Poisson-Lie group. This discussion is superseded by any text on the theory of Poisson-Lie group and affine Poisson-Lie groups (such as $[2,9,10,7])$. In Sect. 2.0 we introduce the notion of pseudodifferential symbols. In 
Sect. 2.1 we prove the main formula that identifies the Poisson Grassmannian with the second Gelfand-Dickey structure. In Sect. 2.2 we prove the fact that the Lie algebra of differential operators is a Poisson-Lie subalgebra and find the corresponding bialgebra structure. In two following sections we discuss the periodical and matrix cases of this construction. In Sect. 2.5 we formulate a conjecture on quantization of the described action in the simplest possible case, when differential operators are of order 1 , and in Sect. 2.6 we give some hints what could be the rigorous topological justification of what we do here.

In this paper we are concerned with two different questions. Therefore all the discussion is divided in two parts: the first part is purely algebraic, the second part is concerned with the "topologizations" of these results in the infinite-dimensional case. A reader interested only in the purely algebraic picture can skip any discussion of the topology on the manifolds in question. Moreover, we fully understand that the discussion of topology is only sketchy here and hope to continue it elsewhere. The only reason we include the topological discussions into this (otherwise purely algebraic) paper is our thinking that the topological mechanisms we use are quite simple, and they help to add some sense to the formulae we write.

We should note now that one of the interpretations of the results obtained here is that the enormous Lie group $\operatorname{GL}\left(C^{\infty}(\mathbb{R})\right)$ acts on the Gelfand-Dickey Poisson manifold in a Poisson-Lie way. From this point of view this is a generalization of the fact that was observed first by M. Semenov: the dressing action of Volterra operators is of Poisson-Lie nature [10]. Indeed, Volterra operators form a Poisson-Lie subgroup in $\operatorname{GL}\left(C^{\infty}(\mathbb{R})\right)$, therefore the action of this subgroup is also a Poisson-Lie action.

\section{Classical r-Matrices and Poisson-Lie Structures}

1.1. The Classical Yang-Baxter Equation. Let us recall what is the classical YangBaxter equation. Let $r \in \mathfrak{g} \otimes \mathfrak{g}$, where $\mathfrak{g}$ is a Lie algebra. We call $r$ a classical $r$-matrix if $r$ satisfies the equation

$$
\left[r^{12}, r^{13}\right]+\left[r^{12}, r^{23}\right]+\left[r^{13}, r^{23}\right]=0 .
$$

Here for a tensor $x \in \mathfrak{g} \otimes \mathfrak{g}$ we denote by $x^{i j}$ the tensor $\sigma_{(12)} x \otimes \mathrm{id} \in$ $U \mathfrak{g} \otimes U \mathfrak{g} \otimes U \mathfrak{g},\left({ }_{i j}^{1}\right) \in \mathfrak{6}_{3}$ being any transmutation of three elements sending 1 to $i$, and 2 to $j, \sigma$ being the standard action of $\boldsymbol{5}_{n}$ on $\underbrace{V \otimes V \otimes \cdots \otimes V}_{n \text { times }}$, and $U \mathfrak{g}$ being the universal enveloping algebra. The bracket $[$,$] denotes the Lie bracket in the Lie$ algebra $U \mathfrak{g} \otimes U \mathfrak{g} \otimes U \mathfrak{g}$ (of course, the image of this bracket in the above formula lies in $\mathfrak{g} \otimes \mathfrak{g} \otimes \mathfrak{g}$ ).

There are several different objects called by the name Yang-Baxter equation. We use this name for Eq. (1.1) and call a solution of this equation a classical $r$-matrix. However, in what follows the $r$-matrices with a particular symmetry property will play a special role, so we need to study the symmetry properties for the left-hand side of the Yang-Baxter equation. Let YB(r) denote the left-hand side of the Yang-Baxter equation. This is a quadratic form in an argument $r$; let us denote the corresponding symmetric bilinear form as $[r, s]$,

$$
[,]:(\mathfrak{g} \otimes \mathfrak{g}) \otimes(\mathfrak{g} \otimes \mathfrak{g}) \rightarrow \mathfrak{g} \otimes \mathfrak{g} \otimes \mathfrak{g}, \quad[r, r]=\mathrm{YB}(r)
$$


Claim 1.1. If $r$ is skewsymmetric $\left(r \in \Lambda^{2} \mathfrak{g}\right)$, then $\mathrm{YB}(r)$ is also skewsymmetric $\left(Y B(r) \in \Lambda^{3} \mathfrak{g}\right)$.

Proof. First of all, we can note that if $\sigma_{(12)} r=\alpha_{1} r, \sigma_{(12)} s=\alpha_{2} s$, (here $\alpha_{1,2} \in\{ \pm 1\}$ ) then $\sigma_{(13)}[r, s]=-\alpha_{1} \alpha_{2}[r, s]$. Really, $\sigma_{(13)}$ changes the first and the last summand of the formula (1.1) (this, of course, does not change any signs), changes the order of terms inside brackets [,] (this changes the sign of the bracket), and changes the order of indices for $r$ (this multiplies one term by $\alpha_{1}$, another by $\alpha_{2}$ ).

Second, the action of $\sigma_{(12)}$ on $[r, r]$ can be described in the similar way: on the first two summands it acts by interchanging them and interchanging the indices for the first occurrence of $r$; on the last summand it acts by interchanging the arguments of the bracket. Both these descriptions result in the change of the sign.

Hence two generators of $\mathfrak{6}_{3}$ act on $\mathrm{YB}(r)$ in the same way as they act on $\Lambda^{3} \mathfrak{g}$. Hence $\mathrm{YB}(r) \in \Lambda^{3} \mathfrak{g}$.

Now we can compare the Yang-Baxter equation with another quadratic equation on an element of $\Lambda^{2} \mathfrak{g}$, the Jacobi equation on Poisson brackets.

1.2. Poisson Manifolds. Let $\eta$ be a 2-vector field on a manifold $X$, i.e., a section of $\Lambda^{2} T X$. Then we can define a skewsymmetric bracket on functions on the manifold $X$ :

$$
(f, g) \mapsto\{f, g\},\left.\quad\{f, g\}\right|_{x}=\left\langle\left.\eta\right|_{x},\left.\left.d f\right|_{x} \wedge d g\right|_{x}\right\rangle .
$$

Here $\langle$,$\rangle denotes the pairing between \Lambda^{2} T_{x} X$ and $\Lambda^{2} T_{x}^{*} X$. Now we can consider the mapping

$$
\operatorname{Jac}(\eta):(f, g, h) \mapsto \underset{f, g, h}{\operatorname{Alt}}\{\{f, g\}, h\},
$$

here Alt denotes the alternation operation. This mapping measures the degree of non-Jacobi-property for the bracket $\{$,$\} .$

It is easy to see that $\operatorname{Jac}(\eta)(f, g, h)$ depends only on 1-jet of the function $f$ and vanishes if $f$ is a constant function. Hence $\left.\operatorname{Jac}(\eta)\right|_{x}$ is, in fact, a mapping

$$
\Lambda^{3} T_{x}^{*} X \rightarrow \mathbb{C},
$$

therefore $\operatorname{Jac}(\eta)$ corresponds to some section $S N(\eta)$ of $\Lambda^{3} T X$ :

$$
\left.\operatorname{Jac}(\eta)(f, g, h)\right|_{x}=\left\langle S N(\eta),\left.\left.\left.d f\right|_{x} \wedge d g\right|_{x} \wedge d h\right|_{x}\right\rangle .
$$

Therefore we have defined a quadratic mapping

$$
S N: \Gamma\left(\Lambda^{2} T X\right) \rightarrow \Gamma\left(\Lambda^{3} T X\right): \eta \mapsto S N(\eta) .
$$

This mapping ${ }^{3}$ is called the Schouten-Nijenhuis bracket. It is quadratic in $\eta$ and is a differential operator of the first order in $\eta$ (we mean that the value of $S N(\eta)$ in $x$ depends only on 1 -jet of $\eta$ in $x$ ).

\footnotetext{
${ }^{3}$ More explicitly, the symmetrical bilinear mapping that corresponds to this quadratic mapping
} 
Remark 1.1. Let us extend the commutator operator from vector fields to the multi-vector fields in a term-by-term way

$$
\begin{aligned}
{\left[v_{1}\right.} & \left.\wedge v_{2} \wedge \cdots \wedge v_{n}, w_{1} \wedge w_{2} \wedge \cdots \wedge w_{m}\right] \\
=\sum_{i, j}(-1)^{i+j}\left[v_{i}, w_{j}\right] & \wedge v_{1} \wedge \cdots \wedge \hat{v}_{i} \wedge \cdots \wedge v_{n} \wedge w_{1} \wedge \cdots \\
& \wedge \hat{w}_{j} \wedge \cdots \wedge w_{m} .
\end{aligned}
$$

In fact it is a supercommutator if we define the degree of $v_{1} \wedge v_{2} \wedge \cdots \wedge v_{n}$ as $n-1$. The commutator of a vector field and a multivector field is the usual Lie derivative action, it is a generalization of this notion. Now it is easy to see that the Schouten-Nijenhuis bracket is a square of a bivector field:

$$
S N(\eta)=[\eta, \eta] \text {. }
$$

In a local coordinate frame we can express $S N(\eta)$ as

$$
S N(\eta)^{i j k}=\underset{i j k}{\operatorname{Alt}} \eta^{i l} \eta_{, l}^{j k}
$$

We use here the tensor notation: $t_{, i}$ for a component $t$ of a tensor denotes the derivative of this component in the direction of the index $i$.

Definition 1.1. A Poisson manifold is a pair of a manifold $X$ and a bivector field $\eta$ on $X$ such that the corresponding bracket $\{$,$\} on functions satisfies the Jacobi identity.$

Further in this paper we often describe Poisson brackets by the corresponding Hamiltonian mappings $T_{x}^{*} X \rightarrow T_{x} X$. This is just the image of the bivector under the identification $\Lambda^{2} T_{x} X$ with a subspace in $\operatorname{Hom}\left(T_{x}^{*} X \rightarrow T_{x} X\right)$.

1.3. Skewsymmetric $r$-Matrices. Let $\eta \in \Lambda^{2} \mathrm{~g}$. Then $\eta$ determines a left-invariant section $\tilde{\eta}$ of $\Lambda^{2} T G, G$ being the group corresponding to the Lie algebra $\mathfrak{g}$. The 3 -vector $S N(\tilde{\eta})$ is, evidently, left-invariant, hence it corresponds to an element $\mathrm{SN}(\eta) \in \Lambda^{3} \mathfrak{g}$. A question arises naturally to compare two elements of $\Lambda^{2} \mathfrak{g}, Y B(\eta)$ and $\mathrm{SN}(\eta)$. For this we can use Eq. (1.3) in the exponential coordinate system. It is easy to see that the derivative (in this coordinate system) at the unity of a leftinvariant tensor $t$ in the direction of vector $X \in \mathfrak{g}$ is proportional to ad $X \cdot t$. Let $c_{j k}^{i}$ be the structure constants for $\mathfrak{g}$ :

$$
\left[X_{j}, X_{k}\right]=c_{j k}^{i} X_{i} \text {. }
$$

Then we can describe $\mathrm{SN}(\eta)$ up to a multiplicative constant as

$$
\underset{i j k}{\operatorname{Alt}} \eta^{i l} \eta_{, l}^{j k}=\underset{i j k}{\operatorname{Alt}}\left(\eta^{i l} c_{l m}^{j} \eta^{m k}+\eta^{i l} c_{l m}^{k} \eta^{j m}\right)
$$

Now it is easy to see that the first summand in parentheses coincides with the term $\left[r^{12}, r^{23}\right]$, the second coincides with the term $\left[r^{13}, r^{23}\right]$ from the formula (1.1), if $r=\eta .^{4}$ Hence the alternation of the expression (1.1) is proportional to the expression (1.4). However, the expression (1.1) is already skewsymmetric if $\eta$ is

${ }^{4}$ Unfortunately, the indices denote two different operations in (1.4) and (1.1) 
skewsymmetric. Therefore Eq. (1.1) is equivalent to the bivector $\eta$ being a Poisson bracket.

Now we can seen that skewsymmetric classical $r$-matrices for Lie algebra $g$ are "the same" as left-invariant Poisson brackets on the corresponding group $G$. Let us consider one such bracket $\tilde{\eta}$. The action of the group $G$ by left multiplication $g \mapsto L_{g}$ preserves the tensor field $\tilde{\eta}$. However, the action of the group $G$ by right multiplications $g \mapsto R_{g}$ does not preserve this tensor field. We want to describe the change of this bivector field under the action of the right multiplication.

Let us consider the bivector field $\tilde{\eta}^{g}=\tilde{\eta}-R_{g *} \tilde{\eta}$ on $G$, where $g \in G$ is fixed. Of course, one possible description is as of a left-invariant bivector field corresponding to a bivector $\eta-\operatorname{Ad}\left(g^{-1}\right) \eta$ at $e \in G$. However, we can look on this field in another way. A left-invariant vector field on $G$ corresponds to the right action of the Lie algebra $g$. Therefore the left-invariant bivector field corresponds to the "action" of a bivector on $G$. From the other side, the bivector field we consider is the result of an action of the element $g \in G$. This can motivate a consideration of the bivector at $g \in G$ that is a value of this bivector field.

Therefore let us identify the Lie algebra with a tangent space to $G$ at $g$ (let us recall that $g$ is fixed) in a left invariant way. As before, we can consider a value $\eta^{(g)} \in \Lambda^{2} T_{g} G$ of the field $\tilde{\eta}^{g}$. Now we claim the compatibility condition between the right action of $g \in G$ and the bivector $\eta^{(g)}$ :

$$
\left.\Lambda^{2}\left(\frac{\partial R_{g}}{\partial g}\right)\right|_{h, g} \cdot \eta^{(g)}=\left.\tilde{\eta}^{g}\right|_{h g} .
$$

Here we consider $R_{g}: h \mapsto h g$ as a function of two variables. The mapping $\left.\Lambda^{2}\left(\frac{\partial R_{g}}{\partial g}\right)\right|_{h, g}$ sends $\Lambda^{2} T_{g} G$ to $\Lambda^{2} T_{h g} G$, since $\left.\left(\frac{d R_{g}}{d g}\right)\right|_{h, g}$ sends $X \in T_{g} G$ to
$\frac{d}{d \varepsilon} h(g+\varepsilon X) \in T_{h g} G$.

Now we can describe the identity

$$
\Lambda^{2}\left(\frac{d R_{g}}{d g}\right) \cdot \eta^{(g)}=\tilde{\eta}^{g}=\tilde{\eta}-R_{g^{*}} \tilde{\eta}
$$

as saying that the map $m: G \times G \rightarrow G:(g, h) \mapsto g h$ is a Poisson map with respect to the bivector fields $\tilde{\eta}$ on the first and the third occurrence of $G$ and the bivector field $g \mapsto \eta^{(g)} \in \Lambda^{2} T_{g} G$ on the second. That means that the inverse image $m^{*}$ is in accordance with the Poisson brackets on the image and the preimage, i.e.,

$$
\left\{m^{*} f, m^{*} g\right\}_{1}=m^{*}\{f, g\}_{2} \text {. }
$$

Here the bracket $\{,\}_{1}$ on $G \times G$ is a sum of brackets on the components (the bracket $\tilde{\eta}$ on the first and the bracket $\eta^{(g)}$ on the second), and the bracket $\{,\}_{2}$ is a bracket $\tilde{\eta}$ on $G$.

This is usually expressed by the words the group G acts in a Lie-Poisson way by right multiplication. These words mean that there is a remarkable relation on the bivector field $\eta^{(g)}$ itself (that it determines the structure of a Poisson-Lie group), and the above compatibility condition between $\eta^{(g)}$ and a change of $\tilde{\eta}$.

Definition 1.2. A group $G$ with a Poisson structure $\eta$ is called a Poisson-Lie group, if the multiplication map $G \times G \rightarrow G$ is compatible with the Poisson brackets and the inversion map $G \rightarrow G$ multiplies the Poisson bracket by -1 . 
Definition 1.3. A Poisson-Lie group $G$ acts on a Poisson manifold $X$ in a PoissonLie way if the action map $X \times G \rightarrow X$ preserves the Poisson brackets.

Remark 1.2. An example of such objects can be found in Sect. 2.1.

Formally, to use this expression we should show first that the bivector field $g \mapsto \eta^{(g)} \in \Lambda^{2} T_{g} G$ on $G$ determines a Poisson structure on $G$ that is odd with respect to inversion on the group and that the map $G \times G \rightarrow G$ is a Poisson map with respect to structures $\eta^{(g)}$ on any occurrence of $G$. As above, we can rewrite the last condition as the condition of the right action of $G$ on itself being a Poisson-Lie action, or that the change of the bivector field with respect to the right action is compatible with the bivector field. But this is a trivial consequence of what is already proven. Really,

$$
\eta^{(g)}=\left(\Lambda^{2} L_{g}\right) \eta-\left(\Lambda^{2} R_{g}\right) \eta, \quad L_{g}, R_{g}: T_{e} G \rightarrow T_{g} G .
$$

However, the first summand is nothing else as the bivector field $\tilde{\eta}$. On the other hand, the action by right multiplication preserves the second summand. Therefore a change of the vector field $g \mapsto \eta^{(g)}$ under the right multiplication coincides with the change of the first summand. However, this change is already computed and coincides with the term coming from the second component of $G \times G$.

To prove that the bracket corresponding to $g \mapsto \eta^{(g)}$ is a Poisson bracket, we can note that this bracket is a difference of two Poisson brackets by (1.6). ${ }^{5}$ So it is sufficient to show that

$$
\left[\left(\Lambda^{2} L_{g}\right) \eta,\left(\Lambda^{2} R_{g}\right) \eta\right]=0 \text { or }[\tilde{\eta}, \operatorname{Inv} \tilde{\eta}]=0,
$$

here Inv being the inversion map on the group, and this is easy. Indeed, since the commutator of a left-invariant and a right-invariant vector fields on $G$ is 0 and the Schouten-Nijenhuis bracket is an extension of the commutator on vector fields, the Schouten-Nijenhuis bracket of a left-invariant and a right-invariant bivector fields is also 0.

1.4. The Modified Yang-Baxter Equation. As we have already seen, the skewsymmetric $r$-matrix makes it possible to construct a Poisson-Lie bracket on the group $G$ and a left-invariant Poisson structure on $G$ that is a Poisson torsor ${ }^{6}$ with respect to the action by right multiplication. However, if we are ready to drop some of these properties we can weaken the restrictions on a $r$-matrix. As we will see in the end of Sect. 1.5 the amount we need to drop is quite small.

Let $r=\eta+t$ be a decomposition of a (non-skewsymmetric) matrix from $\mathfrak{g} \otimes \mathfrak{g}$ into skewsymmetric and symmetric components. Then

$$
[r, r]=[\eta, \eta]+2[\eta, t]+[t, t]
$$

and decomposition of 3-tensor with respect to the action of $\sigma_{(13)}$ is, as we have shown in Sect. 1.3, $([\eta, \eta]+[t, t])+2[\eta, t]$, where the second summand is invariant and the first is multiplied on -1 .

\footnotetext{
${ }^{5}$ The second summand is symmetrical to the first under the mapping $g \mapsto g^{-1}$, so should be also Poisson

${ }^{6}$ I.e., the principal homogeneous space
} 
The restriction we want to impose is the ad $G$-invariance of the 2 -vector $t$. In this case

$$
\begin{aligned}
2[\eta, t]= & {\left[\eta^{12}, t^{13}\right]+\left[\eta^{12}, t^{23}\right]+\left[\eta^{13}, t^{23}\right]+\left[t^{12}, \eta^{13}\right]+\left[t^{12}, \eta^{23}\right] } \\
& +\left[t^{13}, \eta^{23}\right] .
\end{aligned}
$$

The invariance of $t$ implies that $\left[\eta^{i j}, t^{j k}\right]+\left[\eta^{i k}, t^{j k}\right]=0$ for any $i, j, k$. Hence the sum of the second and the third summands, as well as the sum of the fourth and the fifth summands, is 0 . However,

$$
\left[\eta^{12}, t^{13}\right]+\left[t^{13}, \eta^{23}\right]=-\left[\eta^{21}, t^{13}\right]-\left[\eta^{23}, t^{13}\right]=0 .
$$

Hence in this case the 3 -tensor is also skewsymmetric with respect to $\sigma_{(13)}$ :

$$
[r, r]=[\eta, \eta]+[t, t] .
$$

The same considerations as above show that $[t, t]$ is skewsymmetric, hence $[r, r]$ is skewsymmetric. If $r$ is an $r$-matrix, then $[\eta, \eta]=-[t, t]$.

Definition 1.4. The equation

$$
[\eta, \eta]=-[t, t]
$$

on a skewsymmetric matrix $\eta \in \Lambda^{2} \mathfrak{g}$ for a fixed invariant symmetric matrix $t \in S^{2} \mathfrak{g}$ is called the modified Yang-Baxter equation. Usually, slightly abusing notation, a solution of this equation is also called an r-matrix.

We conclude that the Schouten-Nijenhuis bracket $S N(\tilde{\eta})$ for $\tilde{\eta}$ can be computed based on the invariant symmetric bilinear form $t$. Though the bivector field $\tilde{\eta}$ does not determine the Poisson bracket, the discussion above concerning the compatibility of brackets with the map of multiplication remains applicable to this case. Moreover:

Claim 1.2. The bivector field $g \mapsto \eta^{(g)}$ on the group $G$ determines a Poisson-Lie structure on $G$.

Remark 1.3. We can note here that for bivector field of the form (1.6) to be a Poisson structure, the Schouten-Nijenhuis brackets $\mathrm{SN}\left(\left(\Lambda^{2} L_{g}\right) \eta\right)$ and $\mathrm{SN}\left(\left(\Lambda^{2} R_{g}\right) \eta\right)$ should coincide. Since one is left-invariant and another is rightinvariant, they should be both $\mathrm{Ad} G$-invariant. That means that $[\eta, \eta]$ should be ad $G$-invariant. If all ad $G$-invariant elements of $\Lambda^{3} \mathrm{~g}$ can be expressed as $[t, t]$ for some invariant symmetric element $t \in S^{2} \mathrm{~g}$, then the modified Yang-Baxter equation is equivalent to the definition of the Poisson-Lie group with the restriction (1.6).

As a result for a given solution of the modified Yang-Baxter equation we got the Poisson-Lie structure on the group $G$ and a compatibility left-invariant bivector field on the torsor for $G$ with respect to right multiplication. The Schouten-Nijenhuis bracket of this bivector with itself is a left-invariant (and right-invariant) 3 -vector field determined by the given symmetric bilinear form.

The usual way to construct such a solution is to consider a decomposition of a Lie-algebra with an invariant non-degenerated symmetric pairing $t^{*}$ into a direct sum of two Lie subalgebras that are both isotropic with respect to this form, 
$\mathfrak{g}=\mathfrak{a} \oplus \mathfrak{b}$. Since the skew pairing

$$
r^{*}\left(\left(a_{1}, b_{1}\right),\left(a_{2}, b_{2}\right)\right) \stackrel{\text { def }}{=}\left(\left(-a_{1}, b_{1}\right),\left(a_{2}, b_{2}\right)\right)
$$

(where outer parenthesis in the right-hand side denote the scalar multiplication) is skewsymmetric, it defines (by $t^{*}$-duality) a skewsymmetric $r$-matrix.

A very similar way to do it is to consider a decomposition of a semi-simple Lie algebra

$$
\mathfrak{g}=\mathbf{n}_{-} \oplus \mathfrak{h} \oplus \mathbf{n},
$$

and a bracket

$$
\left[\left(a_{1}, b_{1}, c_{1}\right),\left(a_{2}, b_{2}, c_{2}\right)\right] \stackrel{\text { def }}{=}\left(\left(-a_{1}, c_{1}\right),\left(a_{2}, c_{2}\right)\right) .
$$

1.5. Homogeneous Spaces with Poisson-Lie Action. Let us return first to the situation in Sect. 1.3. There we had a Poisson structure on a torsor for $G$ with a Poisson-Lie action. Since this Poisson structure is left-invariant, we can push it back to a structure on any factor-space $H \backslash G$ with respect to the action by left multiplication. Really, consider the projection $G \stackrel{\pi}{\rightarrow} H \backslash G$. If $f$ and $g$ are two functions on $H \backslash G$, then their pull-backs to $G$ are $H$-invariant with respect to the left action. Therefore this Poisson bracket is also $H$-invariant, i.e., is a push-back of a function on $H \backslash G$. Hence we can define a bracket $\eta_{H \backslash G}$ on $H \backslash G$, that is, evidently, a Poisson bracket.

The same considerations applied to the right action show that the action of $G$ on $H \backslash G$ by right multiplication remains the Poisson-Lie action: the map

$$
\left(H \backslash G \times G, \eta_{H \backslash G} \times \eta^{g}\right) \rightarrow\left(H \backslash G, \eta_{H \backslash G}\right)
$$

is a map of Poisson manifolds.

Hence on any right homogeneous space $X$ for $G$ we can construct a Poisson structure on which this Poisson-Lie group acts in a Poisson-Lie way. (It is easy to see that this Poisson structure does not depend on the choice of the basic point on $X$ used for the identification of $X$ and $H \backslash G$. In the point $x \in X$ the bivector $\left.\eta\right|_{x}$ is the image of $r$ under the map

$$
\Lambda^{2} \mathfrak{g} \rightarrow \Lambda^{2}\left(\mathfrak{g} / \mathfrak{g}_{x}\right)=\Lambda^{2} \mathscr{T}_{x} X
$$

where $\mathfrak{g}_{x}=\operatorname{Stab} x \subset \mathfrak{g}$.)

Let us return now to the situation in Sect. 1.4. Let $r$ be a solution of the modified Yang-Baxter equation. Then we can, as above, define on any right $G$-homogeneous space a bivector field satisfying the condition of compatibility with the action of the Poisson-Lie group. However, this bivector field should not necessarily correspond to a Poisson structure.

Nevertheless, in many important cases this form is a Poisson structure. To investigate these cases, we can note that consideration of the space $H \backslash G$ results in restriction of the 3 -vector $[\eta, \eta] \in \Lambda^{3} \mathfrak{g}$ to a quotient space $\Lambda^{3}(\mathfrak{g} / \mathfrak{h})$. Hence if a Lie subalgebra $\mathfrak{h} \subset \mathfrak{g}$ satisfies the condition

$$
\operatorname{Im}([t, t]) \text { in } \Lambda^{3}(\mathfrak{g} / \mathfrak{h}) \text { is } 0,
$$


then any $r$-matrix associated with $t$ results in the true Poisson structure on the corresponding homogeneous space $H \backslash G$ that is compatible with the Poisson-Lie action of $G$.

In particular, if $G$ is semi-simple, then any homogeneous space with a parabolic stabilizer of a point has a Poisson structure associated with any solution of the modified Yang-Baxter equation. In fact it is sufficient that a stabilizer contains a maximal nilpotent subgroup.

Example 1.1. (A Grassmannian Manifold). Let us consider a Grassmannian manifold $\mathrm{Gr}_{k}(V)$ considered as $\mathrm{GL}(V) / M$, where $M=\operatorname{Stab} W_{0}, W_{0} \subset V$. We can identify the tangent space $T_{W} \mathrm{Gr}_{k}\left(\mathbb{C}^{n}\right)$ (here $\left.W \subset \mathbb{C}^{n}\right)$ with $\operatorname{Hom}(W, V / W)$ and cotangent space $T_{W}^{*} \operatorname{Gr}_{k}\left(\mathbb{C}^{n}\right)$ with $\operatorname{Hom}(V / W, W)$, if we consider a pairing

$$
(\alpha, \beta) \mapsto \operatorname{Tr} \alpha \circ \beta, \quad \alpha \in \operatorname{Hom}(V / W, W), \quad \beta \in \operatorname{Hom}(W, V / W) .
$$

Under these identifications the Poisson structure $\eta_{\mathrm{Gr}}$ associated with an $r$ matrix $r \in \Lambda^{2} \mathrm{gl}(V)$ can be expressed via the corresponding Hamiltonian mapping

$$
\begin{aligned}
& \tilde{\eta}_{\mathrm{Gr}}: T_{W}^{*} \operatorname{Gr}_{k}\left(\mathbb{C}^{n}\right) \rightarrow T_{W} \operatorname{Gr}_{k}\left(\mathbb{C}^{n}\right):, \\
& \tilde{\eta}_{\mathrm{Gr}}:\left.\alpha \mapsto \tilde{r}(\alpha)\right|_{W / W} \in \operatorname{Hom}(W, V / W), \quad \alpha \in \operatorname{Hom}(V / W, W) .
\end{aligned}
$$

Here $\tilde{r}$ is the image of $r$ under the standard identification $\mathfrak{g l}(V)^{\otimes 2} \supset \Lambda^{2} \mathrm{gl}(V)$ with End $(\mathfrak{g l}(V))$, for $\beta \in$ End $V$ we denote the corresponding mapping $W \rightarrow V / W$ as $\left.\beta\right|_{W / W}$.

Proof. Consider a tangent vector $X$ at $e \in \mathrm{GL}(V)$. We can pull the corresponding right-invariant vector field to $\mathrm{Gr}_{k}(V)$. It is easy to see that the corresponding tangent vector at $W \in \operatorname{Gr}_{k}(V)$ is $\left.X\right|_{W / W}: W \rightarrow V / W$. (Hence we consider $X$ as a mapping $V \rightarrow V$.)

Now we can consider $\alpha \in \operatorname{Hom}(V / W, W)=T_{W}^{*} \mathrm{Gr}_{k}\left(\mathbb{C}^{n}\right)=T_{W}^{*} \mathrm{GL}(V) / M$ and the corresponding mapping $\alpha^{\prime}: V \rightarrow V$. The above argument shows that the right translation $R_{g-1}^{*} \pi^{*} \alpha$ of the corresponding cotangent vector $\pi^{*} \alpha \in T_{g}^{*} \mathrm{GL}(V)$ (here $g W_{0}=W$ ) is exactly $\alpha^{\prime}$ (if we identify $\mathrm{gl}^{*}$ with $\mathfrak{g l}$ ).

Now the Hamiltonian mapping on the GL sends the corresponding to $\alpha$ rightinvariant covector field to the corresponding to $\tilde{r}(\alpha)$ right-invariant vector field.

A different approach to a definition of a Poisson structure on a parabolic factor can be found in paper [7]. We should note that approach results in a different Poisson structure.

Remark 1.4. As parabolic quotients go, the Poisson Grassmannians satisfy a remarkable property. A choice of $r$-matrix for $\mathrm{gl}(V)$ of standard form (1.9) is given by a Cartan subgroup $H$, i.e., a basis in $V$, and an ordering of this basis (that gives us two opposite Borel subgroups). It is easy to check that the Poisson structure on, say, the flag variety essentially depends on the choice of this ordering. However, the Poisson structures on the Grassmannians are the same for two cyclically permuted orderings.

Proof. Indeed, if we consider an $r$-matrix for $\mathfrak{g l}$ as a mapping $\tilde{r}$ : $\mathfrak{g l} \rightarrow \mathfrak{g l}$, two $r$-matrices corresponding to two cyclically permuted orderings differ by a commutation mapping

$$
\tilde{r}_{1}-\tilde{r}_{2}: \mathfrak{g l}(V) \rightarrow \mathfrak{g l}(V): X \mapsto[z, X]
$$


for an appropriate $z \in$ End $V$. Therefore if $\alpha, \beta \in \operatorname{Hom}(V / W, W)$ are two covectors on $\mathrm{Gr}_{k}(V)$ at $W \in \mathrm{Gr}_{k}(V)$, the difference of values of two Poisson structures on the Grassmannian can be expressed as

$$
\left\langle\tilde{\eta}_{\mathrm{Gr}, 1}-\tilde{\eta}_{\mathrm{Gr}, 2} \alpha \wedge \beta\right\rangle=\operatorname{Tr} \beta \circ[z, \alpha]=\operatorname{Tr}[\alpha, \beta] z .
$$

However, $[\alpha, \beta]=0$ (this means, in fact, that the radical of the stabilizer is commutative), since already $\alpha \beta=\beta \alpha=0$.

This shows as well that the other parabolic spaces with the same properties could be isotropic Grassmannians of middle dimension in the symplectic or even-dimensional orthogonal space or the isotropic conic in a projectivization of an orthogonal space (dropping some exceptional examples). However, we do not know exact results in these cases.

1.5.1. Poisson-Lie subgroups. A notion of a Poisson-Lie group is so tightly connected with a notion of a Poisson-Lie action that the usual notion of a Lie subgroup and Lie subalgebras are useless in this context: the restriction of a Poisson-Lie action on a subgroup usually is not a Poisson-Lie action. To define the additional condition on a subalgebra we need the definition of the Lie algebra structure on the dual space to a Poisson-Lie algebra (i.e., a tangent space to a Poisson-Lie group at $e \in G$ ).

Definition 1.5. Consider a Poisson-Lie group $(G, \eta)$. A Poisson bracket $\{f, g\}$ of any two functions on $G$ vanishes at $e$, and the linear part of this bracket in $e$ is uniquely determined by linear parts of $f$ and $g$ at $e$, if $f(e)=g(e)=0$. This determines a Lie algebra structure on $\mathrm{g}^{*}$.

Definition 1.6. A Lie subalgebra $\mathfrak{h} \subset \mathfrak{g}$ is called a Poisson-Lie subalgebra if its orthogonal complement $\mathfrak{h}^{\perp} \subset \mathfrak{g}^{*}$ is an ideal in $\mathfrak{g}^{*}$. A connected Lie subgroup $H \subset G$ is called a Poisson-Lie subgroup if its Lie algebra is a Poisson-Lie subalgebra.

Lemma 1.1. A Poisson-Lie subgroup is a Poisson submanifold (i.e., a union of symplectic leaves). The restricted Poisson structure makes it a Poisson-Lie group.

Claim 1.3. A restriction of a Poisson-Lie action on a Poisson-Lie subgroup is a Poisson-Lie action.

\section{Gelfand-Dickey Brackets}

The topics of this section are inspired by the paper [8] where an action of the Lie algebra $\mathscr{D}$ of differential operators of an arbitrary order on the space $\mathscr{D}_{n}$ of differential operators of degree $n$ with the leading term $\frac{d^{n}}{d x^{n}}$ (i.e., on the GelfandDickey space) is defined.

Definition 2.1. Denote by $\mathscr{D}$ the Lie algebra of differential operators

$$
\sum_{k=0}^{K} f_{k}(x)\left(\frac{d}{d x}\right)^{k}
$$

on the line. 
Definition 2.2. Let $\mathscr{D}_{n}$ denote the set of differential operators of order $n$ with the leading coefficient 1 :

$$
\left(\frac{d}{d x}\right)^{n}+\sum_{k=0}^{n-1} f_{k}(x)\left(\frac{d}{d x}\right)^{k} .
$$

In the above definitions we did not specify restrictions on the coefficient of differential operators. In what follows we will consider two important cases: in the first (the case of a line) we allow any (smooth) coefficients, in the second (the case of a circle) we consider only periodic coefficients.

As it was shown by Radul, the mentioned action is a Poisson-Lie action, if we consider the second Gelfand-Dickey Poisson structure on the space $\mathscr{D}_{n}$, and the standard bialgebra structure on the Lie algebra $\mathscr{D}$. This bialgebra structure is associated with the structure of the Lie algebra on the linear space $\mathscr{D}^{*}$ arising from the isomorphism of this space and the space of pseudodifferential symbols of negative order.

2.0. The Second Gelfand-Dickey Bracket on the Set of Differential Operators. To define the isomorphism above let us define the space of pseudodifferential symbols (YDS) as an algebra of formal sums

$$
f(x, \xi)=\sum_{-\infty<k \leqq N} f_{k}(x) \xi^{k}
$$

with the composition rule

$$
f(x, \xi) \circ g(x, \xi)=\sum_{n=0}^{\infty} \frac{1}{n !}\left(\frac{\partial}{\partial \xi}\right)^{n} f(x, \xi)\left(\frac{\partial}{\partial x}\right)^{n} g(x, \xi)
$$

and a function $\widetilde{T r}$ on 1-periodical pseudodifferential symbols as

$$
\widetilde{\operatorname{Tr}}\left(\sum_{k=-\infty}^{N} f_{k}(x) \xi^{k}\right)=\int f_{-1}(x) d x .
$$

The definition of $\widetilde{T r}$ has sense in the periodical case (where the integral is taken along the period) or if the coefficients of the pseudodifferential operator are rapidly decreasing. The basic property of $\widetilde{T r}$,

$$
\widetilde{\operatorname{Tr}}(f \circ g-g \circ f)=0,
$$

is the main reason for the notation we use. ${ }^{7}$

It is easy to see that the mapping $\frac{d}{d x} \mapsto \xi$ extends to the mapping from the algebra of differential operators into the algebra of pseudodifferential symbols. Hence we can change the letter $\xi$ in the notation of a pseudodifferential symbol to $\frac{d}{d x}$ without a risk of misinterpretation.

Now the formula

$$
(f, g) \mapsto \widetilde{\operatorname{Tr}}(f \circ g)
$$

${ }^{7}$ Another connection between the usual trace and the function $\widetilde{T r}$ is given by $(2.7)$ 
defines a symmetric bilinear form on the vector space of pseudodifferential symbols. It is easy to see that this form is nondegenerate. Using this form we can identify the dual space to the space of differential operators with a space of pseudodifferential operators of degree $\leqq-1$. Here again there is no problem in the periodical case, and in the case of a line we define a pairing between the vector space of all $\Psi D S$ and the vector space of $\Psi D S$ with compact support.

However, in a usual sense the dual space to a space of smooth functions is the space of generalized functions. Therefore the above formula identifies the dual space to differential operators with the space of $\Psi D S$ of negative order with generalized functions as coefficients. We are going to give a sketch of an explanation of how "to put this difficulty under the carpet" later, in Sect. 2.6. Here we consider only the linear functionals that correspond to $\Psi D S$ with smooth coefficients. Moreover, we pretend that any linear function on the space of $\Psi D S$ corresponds to such a functional. This a usual difficulty in trying to define an infinite-dimensional smooth manifold.

Now consider a cotangent vector to $\mathscr{D}_{n} \subset \mathscr{D}$. The dual space to a subspace is a quotient of the space dual to the ambient space, and we can easily see that this covector corresponds to an element of $\Psi D S_{\leqq-1} / \Psi D S_{\leqq-n-1}$. Below we abuse our notations and call this element of the quotient a symbol too.

Let $L \in \mathscr{D}_{n}, A$ and $B$ be two cotangent to $\mathscr{D}_{n}$ in the point $L$ covectors, represented by pseudodifferential symbols of negative order. Then the formula

$$
(A, B) \mapsto \widetilde{\operatorname{Tr}}\left(A\left((L B)_{+} L-L(B L)_{+}\right)\right),
$$

(where the subscript + denotes taking the purely differential part of a $\Psi D S$ ) determines a bivector field on $\mathscr{D}_{n}$ that induces (as a very cumbersome calculation shows) a Poisson structure on the manifold $\mathscr{D}_{n}$. In fact in what follows we will anyway give another description of this bivector field that will prove that the defined structure is indeed Poisson.

In the periodic case the existence of the right-hand side is clear, in the case of all functions on a line we can note that the symbols $A$ and $B$ are of finite support (since they represent covectors), hence the right-hand side is of finite support.

Remark 2.1. Let us note that the structure (2.1) can be easily rewritten as

$$
(A, B) \mapsto-\widetilde{\operatorname{Tr}}\left(A\left((L B)_{-} L-L(B L)_{-}\right)\right),
$$

where $M_{-}=M-M_{+}$is an "integral" part of a pseudodifferential operator $M$. In fact to define the second Gelfand-Dickey structure we need both these descriptions: the first one shows that the Hamiltonian mapping is given by the formula

$$
B \mapsto(L B)_{+} L-L(B L)_{+},
$$

therefore the image is a differential operator, the second gives that it is

$$
B \mapsto-\left((L B)_{-} L-L(B L)_{-}\right),
$$

therefore it is a pseudodifferential operator of order $<n$. (And therefore a tangent vector to $\mathscr{D}_{n}$.)

In fact in what follows we use only the following property of this Hamiltonian mapping: it sends $B$ to a differential operator of order $<n$ of the form

$$
L(B L)_{-} \text {- something } \circ L
$$

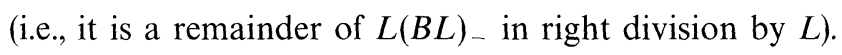


Our task here is to give a description of this Poisson structure based on classical $r$-matrices.

2.1. The Identification with a Grassmannian. Here we define some Poisson structure on a Grassmannian $\mathrm{Gr}_{k}\left(C^{\infty}(\mathbb{R})\right)$ and show the relation between this Poisson structure and the Gelfand-Dickey structure. In turn, to define a Poisson structure on the Grassmannian we need to define an $r$-matrix for the Lie algebra $g l\left(C^{\infty}(\mathbb{R})\right)$. Let us recall that an $r$-matrix for $\mathfrak{g}$ is an element of $\mathfrak{g} \otimes \mathfrak{g}$, therefore we need a definition of tensor square of an infinite-dimensional vector space. As usual in topology, there are some immanent difficulties in such a definition. We postpone the discussion how we can deal with it until Sect. 2.6, and use meanwhile some ad hoc definitions of the objects in question.

So let $V$ be a space of smooth functions on $\mathbb{R}$. We will "take" as $V^{*}$ the space of smooth 1 -forms on $\mathbb{R}$ with a compact support. Hence as $\mathfrak{g l}(V)$ we "can" consider a space of smooth forms $f(x, y) d y$, such that

$$
\forall M \exists N \quad f(x, y)=0 \text { if }|x| \leqq M,|y| \geqq N .
$$

As $g^{l}(V)^{*}$ we "consider" a space of smooth forms $g(x, y) d x$, such that $\exists M f(x, y)=0$ for $|x| \geqq M$. The pairing between these two spaces is

$$
(f(x, y) d y, g(x, y) d x) \mapsto \int_{\mathbb{R} \times \mathbb{R}} f(x, y) g(x, y) d x d y .
$$

As in (1.9) in Sect. 2.2, we can define a symmetric invariant bilinear form on $\mathrm{gl}(V)^{*}$ as

$$
\left(g_{1}(x, y) d y, g_{2}(x, y) d y\right) \stackrel{t}{\mapsto} \int_{\mathbb{R} \times \mathbb{R}} g_{1}(x, y) g_{2}(y, x) d x d y,
$$

and a skewsymmetric bilinear form on $\mathfrak{g l}(V)^{*}$ as

$$
\left(g_{1}(x, y) d y, g_{2}(x, y) d y\right) \stackrel{r}{\mapsto} \int_{\mathbb{R} \times \mathbb{R}} g_{1}(x, y) g_{2}(y, x) \operatorname{sgn}(x-y) d x d y .
$$

The same calculation as in the finite-dimensional case shows that $t+r$ is a solution of the classical Yang-Baxter equation. Now the form (2.3) (being an $r$-matrix) determines a Poisson structure on the space $\mathrm{Gr}_{k}(V)$ and a Poisson-Lie structure on the corresponding group of invertible operators.

Let us emphasize that the above conditions on the supports are absolutely natural and arise from the operators being continuous in the corresponding spaces. The only tricks we did are tricks with smoothness conditions on the kernels.

Remark 2.2. Now, when we have defined the $r$-matrix, we can explain why it cannot be defined as an element of the tensor square of some Lie algebra. Indeed, let us try to extend the definition (2.3) to $g_{1}, g_{2}$ being elements of the space dual to some algebra. Here we consider two cases: the algebras of continuous operators in two spaces of functions on the line, $C^{\infty}(\mathbb{R})$ and $C^{-\infty}(\mathbb{R})$. (The definitions of these topological vector space can be found in Sect. 2.6.)

The Lie algebra $\mathfrak{g}_{1}=\mathfrak{g l}\left(C^{\infty}(\mathbb{R})\right)$ consists of operators with a kernel $f(x, y) d y$ such that the wavefront of $f$ is non-vertical on the $(x, y)$-plane and with the same condition on the kernel as above. Therefore the dual space contains operators with kernels $g(x, y) d y$ such that the wavefront of $g$ is vertical and with the same condition on the support as above. Now in the formula $(*)$ we have a product $g_{1} g_{2}^{\perp}$, 
and the first factor has vertical wavefront, and the second has a horizontal one. Therefore the product is well-defined as a generalized function and has a compact support, therefore the integral $(*)$ and, hence, tensor $t$ is correctly defined. However, in the case of the formula (2.3) we cannot pair this product with $\operatorname{sgn}(x-y)$, since $\operatorname{sgn}(x-y)$ is not a smooth function on a plane!

In the same way if we consider $g_{2}=g l\left(C^{-\infty}(\mathbb{R})\right)$, then we get the same with a change of vertical and horizontal directions. However, if we consider $\mathfrak{g}_{1}^{*} \cap \mathfrak{g}_{2}^{*}$, as in Sect. 2.6, then the wavefront should be both horizontal and vertical, therefore any operator from this set has a smooth kernel, and the product with $\operatorname{sgn}(x-y)$ in formula (2.3) is well-defined.

Theorem 2.1. The defined on $\mathrm{Gr}_{k}(V)$ Poisson structure coincides (on an open subset of $\left.\operatorname{Gr}_{k}(V)\right)$ with two times the second Gelfand-Dickey Poisson structure on the set $\mathscr{D}_{k}$ of differential operators of degree $k$ with the leading term $\left(\frac{d}{d x}\right)^{k}$ under the identification

$$
\mathscr{D}_{k} \ni L \stackrel{s}{\mapsto} W=\{f \mid L f=0\}
$$

Proof. The two Poisson structures we want to equate are both given by their Hamiltonian mappings. However, these Hamiltonian mappings are described in different languages. In the $\Psi D S$ description we use identification of the tangent space with the set of differential operators and of the cotangent space with pseudodifferential operators, in the Grassmannian description we identify them with the space of linear mappings $W \rightarrow V / W$ and $V / W \rightarrow W$ correspondingly. So the first step is to relate these two pairs of identifications. This step takes the longest part of the proof.

First of all we describe which mapping $W \rightarrow V / W$ corresponds to a variation $\delta L$ of a differential operator $L, W=\operatorname{Ker} L$.

Lemma 2.1. Consider the identification of $\mathscr{D}_{k}$ with the subset of the Grassmannian $\mathrm{Gr}_{k}(V)$. Consider a tangent vector to $\mathscr{D}_{k}$ at $L \in \mathscr{D}_{k}$. Let $\operatorname{Ker} L=W$. We have two different representations of this vector: a differential operator $\delta L$ and a mapping $M: W \rightarrow V / W$. Then

$$
M=-\pi \circ L^{-1} \delta L_{\mid W},
$$

where $\pi$ is the projection $V \rightarrow V / W$.

Proof. Let us consider the equation

$$
(L+\delta L)(f+\delta f)=0 .
$$

It means that

$$
\delta f=-L^{-1} \delta L \cdot f .
$$

Hence the corresponding to $\delta L \operatorname{map} W \rightarrow V / W$ is $-\pi \circ L^{-1} \delta L_{\mid W}$, where $\pi$ is the projection $V \rightarrow V / W$. In fact, the map $L^{-1}$ is, of course, undefined, since the map $L$ has a kernel. However, in our case this kernel is exactly $W$ ! Hence the composition $\pi \circ L^{-1}$ is correctly defined. (The map $L$ is obviously a surjection.) Without abusing the notations we can denote $\pi \circ L^{-1}$ as $L^{-1}$. Now we can interpret (2.5) as 
two descriptions of the tangent space being connected by

$$
\delta L \mapsto-L^{-1} \delta L_{\mid W / W}
$$

Here for a mapping $B: V \rightarrow V$ we denote by $B_{\mid W / W}$ the corresponding mapping $W \rightarrow V / W$.

Second, let us describe the relations on the cotangent space.

Lemma 2.2. Consider the identification of $\mathscr{D}_{k}$ with the subset of the Grassmannian $\mathrm{Gr}_{k}(V)$. Consider a cotangent vector to $\mathscr{D}_{k}$ at $L \in \mathscr{D}_{k}$. Let $\operatorname{Ker} L=W$. We have two different representations of this covector: a pseudodifferential symbol $A$ and a mapping $A^{\prime}: V / W \rightarrow W$. Consider $A^{\prime}$ as a finite-dimensional mapping $V \rightarrow V$. Then

$$
A=\text { the symbol of }-\frac{1}{2} \tilde{r}\left(A^{\prime} \circ L^{-1}\right) \text {, }
$$

where $\pi$ is the projection $V \rightarrow V / W$.

Proof. The identification above shows that

$$
\widetilde{\operatorname{Tr}} A \circ \delta L=-\operatorname{Tr} A^{\prime} \circ L^{-1} \delta L
$$

for any $\delta L$. We are going to use this equation to express $A$ in terms of $A^{\prime}$; therefore we need here a relation between functions $\widetilde{T r}$ and $\mathrm{Tr}$. However, this relation is very simple:

$$
\operatorname{Tr} K(x, y) d y=\widetilde{\operatorname{Tr}}\left(\frac{1}{2} \operatorname{sgn}(x-y) K(x, y) d y\right)
$$

for a smooth kernel $K(x, y) d y$. Several words about what is in the right-hand side.

We defined so while only the notion of a pseudodifferential symbol. However, in a similar way we could define a notion of a pseudodifferential operator. It is a sum of a differential operator and of an operator of negative order. In turn an operator of negative order is an operator with a piecewise-smooth kernel $L(x, y) d y$ with the only jump (of the first kind) on the diagonal $x=y$ (as the operator in the right-hand side of (2.7)). It is easy to construct a homomorphism from operators to symbols (this homomorphism is uniquely determined by the fact that it sends the set of operators with smooth kernels to 0 ).

Now it is easy to see that the corresponding to the kernel $\frac{1}{2} \operatorname{sgn}(x-y) K(x, y) d y$ symbol is $K(x, y) \partial^{-1}+O\left(\partial^{-2}\right)$, that proves the above formula. Indeed, if $K(x, y) d y$ is a kernel of a finite-dimensional operator $K: V \rightarrow V$, we can write $\operatorname{Tr} K \stackrel{\text { def }}{=} \operatorname{Tr}\left(\left.K\right|_{\operatorname{Im} K}\right)$ as $\int K(x, x) d x$. This formula extends naturally to operators with smooth kernel and satisfies any natural relation that is valid in the finitedimensional case. In fact we can rewrite (2.7) as

$$
\operatorname{Tr} K=\frac{1}{2} \widetilde{\operatorname{Tr}} \tilde{r}(K)
$$

In what follows we use formula (2.7) not only for smooth $K$, but also for operators of finite rank. The only difference in the proof of this modification is the reference to the wavefront of $K$ being horizontal. 
Hence we can rewrite (2.6) as

$$
\widetilde{\operatorname{Tr}} A \circ \delta L=-\frac{1}{2} \widetilde{\operatorname{Tr}} \tilde{r}\left(A^{\prime} \circ L^{-1} \delta L\right) .
$$

(Since the operator $A^{\prime}$ is of finite rank, the operator in brackets also is of finite rank, therefore we can apply the formula (2.7) to it.)

I claim that $A$ is the symbol of the operator $-\frac{1}{2} \tilde{r}\left(A^{\prime} \circ L^{-1}\right)$. Indeed, if $B$ is an operator with a smooth kernel and $M$ is a differential operator, we can note that kernels of $\tilde{r}(B \circ M)$ and $\tilde{r}(B) \circ M$ coincide outside of the diagonal, therefore the former is an integral part of the latter:

$$
\tilde{r}(B \circ M)=(\tilde{r}(B) \circ M)_{-} .
$$

Therefore values of $\widetilde{\operatorname{Tr}}$ on $\tilde{r}(B \circ M)$ and on $\tilde{r}(B) \circ M$ are the same. We obtain

$$
\widetilde{\operatorname{Tr}} A \circ \delta L=-\frac{1}{2} \widetilde{\operatorname{Tr}} \tilde{r}\left(A^{\prime} \circ L^{-1}\right) \circ \delta L,
$$

which proves the assertion. Now we can write the second translation formula connecting two descriptions of the cotangent space: let a cotangent vector to $\mathscr{D}_{n}$ correspond both to a pseudodifferential symbol $A$ of negative order to an operator $A^{\prime}: V / W \rightarrow W$. Then ${ }^{8}$

$$
A=\text { the symbol of }-\frac{1}{2} \tilde{r}\left(A^{\prime} \circ L^{-1}\right) .
$$

Now we can begin the comparison of the Hamiltonian mappings themselves, since we know the translation formulae between languages they are defined in. The Hamiltonian mapping defined in terms of Grassmannian sends a cotangent vector represented by $A^{\prime}: V / W \rightarrow W$ to the tangent vector to the Grassmannian represented by $\tilde{r}\left(A^{\prime}\right)_{\mid W / W}: W \rightarrow V / W$. From the other side, the pseudodifferential symbol $A$ corresponding to the cotangent vector is already expressed in terms of the pseudodifferential operator $\mathbf{A}=\tilde{r}\left(A^{\prime} \circ L^{-1} \circ L^{-1}\right)$. Therefore we can write $\tilde{r}\left(A^{\prime}\right)$ in terms of $\mathbf{A}$ :

$$
\tilde{r}\left(A^{\prime}\right)=\tilde{r}\left(A^{\prime} \circ L^{-1} \circ L\right)=\left(\tilde{r}\left(A^{\prime} \circ L^{-1}\right) \circ L\right)_{-}=-2(\mathbf{A} \circ L)_{-}
$$

(we used (2.8)). Therefore to find the differential operator $\delta L$ corresponding to $\tilde{r}\left(A^{\prime}\right)_{\mid W / W}$ we need to solve the equation

$$
-L^{-1} \delta L_{\mid W}=-2(\mathbf{A} \circ L)_{-\mid W / W} .
$$

It is equivalent to

$$
\delta L_{\mid W}=2 L \circ(\mathbf{A} \circ L)_{-\mid W} .
$$

Therefore to find $\delta L$ we need to find a differential operator of order $<n$ that coincides with $2 L \circ(\mathbf{A} \circ L)_{-}$when considered on $W$. Now the same argument as in Remark 2.1 shows that the operator

$$
-2\left((L \mathbf{A})_{-} L-L(\mathbf{A} L)_{-}\right)
$$

\footnotetext{
${ }^{8}$ In fact we cannot claim that two sides of this formula are exactly equal. They are equal mod $\Psi \mathrm{DS}_{\leqq-n-1}$, since a cotangent vector to $\mathscr{D}_{n}$ is an element of $\Psi \mathrm{DS}_{\leqq-1} / \Psi \mathrm{DS}_{\leqq-n-1}$
} 
is a differential operator of order $<n$ and that it coincides with $2 L \circ(\mathbf{A} \circ L)_{-}$when restricted on $W=\operatorname{Ker} L$. Moreover, we can change operators to symbols in this formula, since the result is a differential operator anyway. Therefore the Hamiltonian mapping associated with the Poisson structure on the Grassmannian is indeed a multiple of Hamiltonian mapping for the second Gelfand-Dickey structure under the above identification.

Remark 2.3. It is easy to see that the identification of the theorem sends the space $\mathscr{D}_{k}$ of differential operators to an open subset of the Grassmannian. In fact we have extended the second Gelfand-Dickey structure to a "compactification" of the space $\mathscr{D}_{k}$.

Corollary 2.1. On the space $\mathscr{D}_{k}$ of differential operators with the second GelfandDickey Poisson structure a (local) action of a Poisson-Lie group $\mathrm{GL}\left(C^{\infty}(\mathbb{R})\right)$ is defined.

Corollary 2.2. The bracket (2.1) satisfies the Jacobi identity.

Proof. Indeed, it coincides with the Poisson bracket on the Grassmannian.

\subsection{A Poisson-Lie Algebra of Differential Operators as a Poisson-Lie Subalgebra of} $\mathrm{gl}$ : The previous corollary shows that there is a Poisson-Lie action of an enormous group GL (functions on $\mathbb{R}$ ) on (an extension of) the second Gelfand-Dickey structure. However, as we have already said, A. Radul defined a Poisson-Lie action of a Lie algebra of differential operators on the same space. Since these two actions are compatible, the natural conjecture is that the latter algebra is a Poisson-Lie subalgebra of the Lie algebra of GL (functions on $\mathbb{R}$ ).

Theorem 2.2. The subspace $\mathscr{D}$ of differential operators is a Lie-Poisson subalgebra of the Poisson-Lie algebra gl (functions on $\mathbb{R}$ ). The Poisson-Lie structure on this space coincides with the structure defined in Sect. 2.0.

Proof. We want to prove that the Lie algebra $\mathscr{D}$ of differential operators is a Lie-Poisson subalgebra (in the sense of Sect. 1.5.1) of the Lie algebra of continuous operators in the space of functions on $\mathbb{R}$. It is easy to see that $\mathscr{D}^{\perp} \subset$ $\mathfrak{g l}(\text { functions on } \mathbb{R})^{*}=\{f(x, y) d y\}$ coincides with the space of forms $f(x, y) d y$ that vanish together with any derivative on the diagonal $x=y$.

On the other hand, it is easy to see that the Lie-algebraic structure on the coalgebra gl(functions on $\mathbb{R})^{*}$ is connected with a decomposition of an element $f$ of this coalgebra into a sum of "increasing" and "decreasing" Volterra operators:

$$
\begin{aligned}
f(x, y) d y & =\frac{(\operatorname{sgn}(x-y)+1)}{2} f(x, y) d y+\frac{(-\operatorname{sgn}(x-y)+1)}{2} f(x, y) d y \\
& =f_{+}(x, y) d y+f_{-}(x, y) d y .
\end{aligned}
$$

Using this decomposition we can describe the commutator of two elements $f, g \in \mathfrak{g l}(\text { functions on } \mathbb{R})^{*}$ as

$$
[f, g]=\left[f_{+}, g_{+}\right]-\left[f_{-}, g_{-}\right]
$$

and to determine the bracket on the spaces of increasing (and decreasing) Volterra operators as a usual Lie bracket:

$$
\left[f_{ \pm}, g_{ \pm}\right](x, y) d y=\left(\int\left(f_{ \pm}(x, z) g_{ \pm}(z, y)-g_{ \pm}(x, z) f_{ \pm}(z, y)\right) d z\right) d y \text {. }
$$


A simple calculation shows that these two formulae indeed determine a bracket with values in smooth functions on $\mathbb{R} \times \mathbb{R}$ :

Lemma 2.3. The form $[f, g]$ is smooth.

Proof. It is obvious that the forms $\left[f_{+}, g_{+}\right]$and $\left[f_{-}, g_{-}\right]$are smooth outside of the diagonal and up to it as compositions of Volterra operators with smooth kernels. Hence the form $[f, g]$ can have a jump of the first kind on the diagonal maximum. To prove that it has no jump let us consider a symbol of this kernel considered as a pseudodifferential operator. It is sufficient to show that this symbol is 0 .

Again, for this it is sufficient to show that the operators $\left[f_{+}, g_{+}\right]$and $\left[f_{-}, g_{-}\right]$ $=\left[-f_{-},-g_{-}\right]$have equal symbols. However, the kernels of operators $f_{+}$and $-f$ - differ on a smooth function $f$, hence have equal symbols. Therefore the commutators in question also have equal symbols.

Now it is almost clear that the space of forms $f(x, y) d y$ that have a zero $\infty$-jet on the diagonal form an ideal with respect to this bracket. Indeed, it is sufficient to show that the bracket with, say, a lowering Volterra operator $g_{-}$is such. Now the corresponding kernels $f_{ \pm}$are smooth on the whole $\mathbb{R} \times \mathbb{R}$ including diagonal, hence have zero symbol, hence their brackets with an arbitrary Volterra operator (of the same direction) also have zero symbols, hence are smooth on the whole $\mathbb{R} \times \mathbb{R}$ including the diagonal. Moreover, $\left[f_{+}, g_{-}\right]=0$, and the kernel $\left[f_{-}, g_{-}\right]$is again Volterra, therefore the symbol corresponds to the jet on the diagonal. Therefore the kernel of $\left[f, g_{-}\right]$has a vanishing $\infty$-jet on the diagonal.

Therefore the Lie subalgebra $\mathscr{D}$ of differential operators is indeed a Poisson-Lie subalgebra. To determine the corresponding Poisson bracket on the dual space we should consider a structure of quotient-algebra on

$$
\mathscr{D}^{*}=\operatorname{gl}(\text { functions on } \mathbb{R})^{*} / \mathscr{D}^{\perp} \text {. }
$$

The latter space is a space of $\infty$-jets of functions along the diagonal, and the corresponding Lie-algebraic structure is the quotient-structure of the Lie algebra of (say, increasing) Volterra operators with a smooth up to the diagonal kernel by the ideal of Volterra operators with a smooth everywhere kernel. However, this is nothing else but the algebra of pseudodifferential symbols of an order $\leqq-1$.

Proposition 2.1. The action of differential operator $X \in D$ on the differential operator $L \in \mathscr{D}_{k}$ is given by the left remainder of $L X$ modulo $L$ :

$$
X \cdot L=-L X+Y L,
$$

where $Y \in \mathscr{D}$ is chosen in the way that the right-hand side is of degree $<k$ :

$$
Y=\left(L X L^{-1}\right)_{+} .
$$

Here the subscript + denotes taking the differential part.

Proof. By definition, if $L f=0$, then $(L+\varepsilon X \cdot L)(f+\varepsilon X f)=O\left(\varepsilon^{2}\right)$. This means that $X \cdot L+L X$ is 0 on any function $f$ such that $L f=0$. Therefore $X \cdot L+L X=Y L$ for some $Y$.

2.3. The Periodical Case. We have considered a Poisson structure on the GelfandDickey manifold in the case of operators on the whole line. However, it is known 
that the absolutely parallel theory exists in the case of periodical differential operators. Here we discuss the changes we should include in the definition of the Grassmannian and the group $\operatorname{GL}\left(C^{\infty}(\mathbb{R})\right)$ to use the natural identification of the set of periodical differential operators with a subset of the Grassmannian. Such an identification obviously exists, since the periodical differential operator is automatically a differential operator on a line. We get a natural subset of the Grassmannian corresponding to operators with periodic coefficients, that consists of invariant subspaces with respect to the action of $\mathbb{Z}$ by translations on the line. However, we want to consider this space as a space with an action of some analogue of $\operatorname{GL}\left(C^{\infty}(\mathbb{R})\right)$.

It is easy to see that in this way we can generalize the construction above to the periodical case. The main difficulty is to define the corresponding variant of the group of matrices $\operatorname{GL}\left(C^{\infty}(\mathbb{R})\right)$. However, consider a natural action of the group $\mathbb{Z}$ on $C^{\infty}(\mathbb{R})$ by translations. Denote by $\operatorname{GL}\left(C^{\infty}(\mathbb{R})\right)^{\mathbb{Z}}$ the subgroup of operators that commute with $\mathbb{Z}$. Now if $f(x)$ is a function satisfying the condition $f(x+1)=\lambda f(x), \lambda \neq 0$, then $A f$ also satisfies this relation if $A \in \operatorname{GL}\left(C^{\infty}(\mathbb{R})\right)^{\mathbb{Z}}$. Denote by $\operatorname{Gr}_{k}\left(C^{\infty}(\mathbb{R})\right)^{\mathbb{Z}}$ the subspace of $\operatorname{Gr}_{k}\left(C^{\infty}(\mathbb{R})\right)$ consisting of $\mathbb{Z}$-invariant subspaces in $C^{\infty}(\mathbb{R})$. Say, the Grassmannian $\mathrm{Gr}_{1}$ consists of spaces spanned by functions $f$ satisfying the above condition with an arbitrary $\lambda$. If $V \in \operatorname{Gr}_{k}\left(C^{\infty}(\mathbb{R})\right)^{\mathbb{Z}}$, then the operator of translation can be restricted to $V$. This determines an "invariant" of $V$ that is the conjugacy class of this restriction. In the case of $\mathrm{Gr}_{1}$ this invariant is equal to $\lambda$.

Now the group $\mathrm{GL}\left(C^{\infty}(\mathbb{R})\right)^{\mathbb{Z}}$ acts on the space $\mathrm{Gr}_{k}\left(C^{\infty}(\mathbb{R})\right)^{\mathbb{Z}}$ and this action preserves the above "invariant." It is clear that the orbits of this action are numerated by this invariant.

The dual space to the Lie algebra $\left(\operatorname{gl}\left(C^{\infty}(\mathbb{R})\right)^{\mathbb{Z}}\right)^{*}$ (almost - see above) has a natural decomposition into the sum of the space of upper-triangular (lowering Volterra) operators and lower-triangular (raising Volterra) operators. The corresponding $r$-matrix is nevertheless correctly defined, therefore we can define a structure of the Poisson-Lie group on $\operatorname{GL}\left(C^{\infty}(\mathbb{R})\right)^{\mathbb{Z}}$ and the corresponding Poisson structures on the homogeneous spaces for this group, say, on orbits of action on $\operatorname{Gr}_{k}\left(C^{\infty}(\mathbb{R})\right)^{\mathbb{Z}}$.

Taking the union of these structures, we get a Poisson structure on the whole manifold $\operatorname{Gr}_{k}\left(C^{\infty}(\mathbb{R})\right)^{\mathbb{Z}}$. Consider now the differential operator that corresponds to a subspace $V \in \operatorname{Gr}_{k}\left(C^{\infty}(\mathbb{R})\right)^{\mathbb{Z}}$. It is easy to see that this operator is periodical, therefore we can consider the (periodical case of the) second Gelfand-Dickey Poisson structure on this set. The one-to-one repetition of the above arguments proves the

Theorem 2.3. The Poisson structure on the $\mathbb{Z}$-invariant Grassmannian coincides with the second Gelfand-Dickey Poisson structure on the set of periodical differential operators. There is a (local) Poisson-Lie action of the group $\operatorname{GL}\left(C^{\infty}(\mathbb{R})\right)^{\mathbb{Z}}$ on the latter Poisson manifold.

Corollary 2.3. The differential operators with periodic coefficients form a PoissonLie subalgebra in the bialgebra $\mathrm{gl}\left(C^{\infty}(\mathbb{R})\right)^{\mathbb{Z}}$. The action on the periodic Grassmannian is a Poisson-Lie action.

We gained a lot because we considered not the operators in the space of functions on a circle, but the $\mathbb{Z}$-invariant operators on the line. Indeed, the kernel of the operator of the former type is a "function" on $S^{1} \times S^{1}$ and there is no 
decomposition on the upper- and lower-triangular operators. However, in the case considered above the kernel is a function $K(x, y) d y$ such that

$$
K(x, y)=K(x+1, y+1),
$$

and the decomposition exists.

2.4. The Matrix Case. Here we want to make a little remark that what we have done can be generalized to the case of matrix operators without any problem. Instead of $\operatorname{GL}\left(C^{\infty}(\mathbb{R})\right)$ we should consider $\operatorname{GL}\left(C^{\infty}\left(\mathbb{R}, \mathbb{R}^{n}\right)\right)$, where $C^{\infty}\left(\mathbb{R}, \mathbb{R}^{n}\right)$ is the space of vector-functions on $\mathbb{R}$. Instead of $\mathrm{Gr}_{k}\left(C^{\infty}(\mathbb{R})\right)$ we should consider the Grassmannian $\mathrm{Gr}_{n k}\left(C^{\infty}\left(\mathbb{R}, \mathbb{R}^{n}\right)\right)$. Then instead of the set $\mathscr{D}_{k}$ of differential operators of order $k$ we get the set of differential operators of order $k$ with matrix coefficients of the size $n \times n$.

In the same way as above we get the identification of the second GelfandDickey Poisson structure on the set of matrix differential operators (it is given by the same formula (2.1)) of order $k$ with the Poisson structure on the Grassmannian $\mathrm{Gr}_{k n}\left(C^{\infty}\left(\mathbb{R}, \mathbb{R}^{n}\right)\right)$.

In contrast with the scalar case already the case $k=1$ is very interesting. The set of differential operators in question is

$$
\{\partial+A(x)\}
$$

where $A$ is a matrix function of $x$. Let us consider the periodical case. In this case this set is nothing else but a hyperplane $c=1$ in the dual space to a central extension of the algebra of currents with values in $\mathrm{gl}_{n}$. The Poisson structure of Kirillov-Lie on this leaf coincides with the second Gelfand-Dickey Poisson structure, hence on the hyperplane $c=2$ the Kirillov-Lie Poisson structure coincides with two times this structure. Therefore we get a

Corollary 2.4. There is a natural (local) Poisson-Lie action of the Poisson-Lie group $\operatorname{GL}\left(C^{\infty}(\mathbb{R}, \mathbb{R})\right)^{\mathbb{Z}}$ on a hyperplane $c=2$ of the Kirillov-Lie Poisson structure on the dual space to the matrix current algebra.

Let us consider this action slightly more carefully. We can consider the subgroup of currents in $\operatorname{GL}\left(C^{\infty}(\mathbb{R}, \mathbb{R})\right)^{\mathbb{Z}}$, i.e., of operators of multiplication by a periodic matrix-function. It is easy to check that this action coincides with the coadjoint action of this group on the dual space to the Lie algebra. (It is clear that the central extension acts in a trivial way, so we can consider the coadjoint action of the non-extended group.)

In fact this subgroup is a Poisson-Lie subgroup with the trivial Poisson structure. This is compatible with the fact that the coadjoint action preserves the Kirillov-Lie Poisson structure. Moreover, we can consider the momentum mapping for the action of this subgroup on $\{\partial+A\}$ considered as an abstract Poisson manifold. It is easy to check that this mapping is identical on this manifold. Therefore the action of $\operatorname{GL}\left(C^{\infty}\left(\mathbb{R}, \mathbb{R}^{n}\right)\right)^{\mathbb{Z}}$ on this (Poisson) manifold makes it possible to reconstruct the inclusion of this manifold into the dual space to the Lie algebra.

Moreover, we can also consider the Lie subalgebra of periodic vector fields on the line

$$
\text { Vect } \subset \operatorname{gl}\left(C^{\infty}\left(\mathbb{R}, \mathbb{R}^{n}\right)\right)^{\mathbb{Z}}
$$


This Lie subalgebra is a Poisson-Lie subalgebra of the Lie algebra of periodic differential operators, moreover, the corresponding Lie algebra bracket on the dual space is 0 . (So the situation is similar to the above action of currents.) Therefore the action of this subalgebra is a trivial case of Poisson-Lie action: it preserves the Poisson structure on the Grassmannian.

2.5. A Conjecture on Quantization: The Kac-Moody Case. Let us consider a would-be quantization of Corollary 2.4. The quantization of the hyperplane in the dual space to the Lie algebra is something like the enveloping algebra with a fixed central charge. The quantization of the action of Vect on this hyperplane is a Sugavara inclusion, that gives us an (adjoint) action of the Virasoro algebra on the enveloping algebra of the central extension of the currents algebra. In what follows we consider the Sugavara inclusion as an inclusion into the space of (inner) derivatives of this universal enveloping algebra.

Let us look on a possible quantization of the action of $\mathfrak{g l}\left(C^{\infty}\left(\mathbb{R}, \mathbb{R}^{n}\right)\right)^{\mathbb{Z}}$. This should be an action of some big algebra on the enveloping algebra, but not by differentials, as in the case of Sugavara inclusion (where differentials were inner), but by transformations changing the multiplication law. (The Sugavara inclusion acts by differential only because the action of Vect preserves the Poisson structure, which, in turn, is explained by the trivial Lie algebra structure on Vect*). Indeed, the quantization of

$$
G \times X \rightarrow X
$$

is (on the language of deformations $\mathcal{O}_{q}(X)$ of the algebra $\mathcal{O}(X)$ of functions on $X$ )

$$
\mathcal{O}_{q}(X) \rightarrow \mathcal{O}_{q}(X) \otimes \mathcal{O}_{q}(G),
$$

or

$$
\mathcal{O}_{q}(G)^{*} \otimes \mathcal{O}_{q}(X)^{*} \rightarrow \mathcal{O}_{q}(X)^{*}
$$

(i.e., an action of $\mathcal{O}_{q}(G)^{*}$ in $\left.\mathcal{O}_{q}(X)^{*}\right)$, or, what is the same, an action of $\mathcal{O}_{q}(G)^{*}$ in $\mathcal{O}_{q}(X):$

$$
\mathcal{O}_{q}(G)^{*} \otimes \mathcal{O}_{q}(X) \rightarrow \mathcal{O}_{q}(X) .
$$

If the action of $G$ is local, as above, we should change $G$ to the infinitesimal neighborhood of $e \in G$, which results in a change of $\mathscr{O}_{q}(G)^{*}$ to $U_{q}(\mathfrak{g})$. In our situation we get the following

Conjecture 2.1. There is an action

$$
U_{q}\left(\mathfrak{g l}\left(C^{\infty}\left(\mathbb{R}, \mathbb{R}^{n}\right)\right)^{\mathbb{Z}}\right) \otimes U\left(\widehat{\mathfrak{g l}}_{n, c=2 \varphi(q)}\right) \rightarrow U\left(\widehat{\mathfrak{g l}}_{n, c}=2 \varphi(q)\right)
$$

such that the corresponding mapping

$$
U_{q}\left(\mathfrak{g l}\left(C^{\infty}\left(\mathbb{R}, \mathbb{R}^{n}\right)\right)^{\mathbb{Z}}\right) \otimes U\left(\widehat{\mathfrak{g l}}_{n, c}=2 \varphi(q)\right)^{*} \rightarrow U\left(\widehat{\mathfrak{g l}}_{n, c}=2 \varphi(q)\right)^{*}
$$

is a morphism of coalgebras. Here $\varphi$ is unknown function, $\varphi^{\prime}(1)=1$.

Remark 2.4. Due to usual anomalies, a possible modification is a change of some "obvious" value of $2 \varphi(q)$ to $2 \varphi(q)-h$, where $h$ is the dual Coxeter number. It is interesting to compare this conjecture with the results of [4].

What we constructed in this paper is a prequantization of this action and a generalization of this prequantization to $W_{n}$-algebras. 
2.6. The Topological Approach. In Sects. 2.0 and 2.1 we promised to give an explanation how to modify the definitions of the objects we consider to identify the space of linear functionals on smooth functions with the set of smooth functions itself. Let us recall that in the usual sense this set is the set of generalized functions. However, to define the Poisson structure on the sets we consider we need to restrict these functionals to be smooth functions.

Essentially we say that to avoid this difficulty we should consider a set of smooth functions on the set of pseudodifferential symbols that is much finer than the set of continuous functions. Let us recall that by the usual definition the set of smooth linear functions on a topological vector space $V$ coincides with the set of continuous linear functions $V^{*}$. If we drop this restriction, then we get a much bigger number of possible sheaves of smooth functions. A choice of such object determines a subset of smooth linear functions

$$
V_{\text {smooth }}^{*} \subset V^{*} \text {. }
$$

The dual picture is the inclusion

$$
V \subset V_{\text {antismooth }} \stackrel{\text { def }}{=}\left(V_{\text {smooth }}^{*}\right)^{*}
$$

If $\left(V_{\text {smooth }}^{*}\right)^{* *}=V_{\text {smooth }}^{*}$, and $V^{* *}=V$, then the latter inclusion determines the former. In this case we (by definition) call a function on $V$ a smooth function if it is a restriction on $V$ of a smooth "in the usual sense" function on $V_{\text {antismooth. It is }}$ usually expressed by saying that we consider two different topologies on $V$ (introduction of a different (and weaker) topology automatically adds additional elements to a vector space, therefore the vector space increases to be $V_{\text {antismooth }}$ ).

Therefore we should work in the category of rigged topological vector spaces, i.e., spaces with several fixed topologies. Here we define the inclusion

$$
V \subset V_{\text {antismooth }}
$$

corresponding to the Gelfand-Dickey structure. First of all, if we fix a topology on the space of allowed coefficients, then we can consider on the space of symbols the topology of a direct limit of inverse limits, i.e., if we denote by $\Psi \mathrm{DS}_{m}$ the space of UDS of degree no more than $m$, then

$$
\Psi D S=\text { ind } \lim _{N \rightarrow \infty} \text { proj } \lim _{M \rightarrow \infty} \Psi \mathrm{DS}_{N} / \Psi \mathrm{DS}_{-M} .
$$

Since the space $\Psi \mathrm{DS}_{N} / \Psi D \mathrm{~S}_{-M}$ is a finite product of spaces of coefficients, fixation of a topology on smooth functions fixes a topology on $\Psi D S$. Now we consider on the space of functions on the line a pair of topologies:

$$
C^{\infty}(\mathbb{R}) \subset C^{-\infty}(\mathbb{R}) .
$$

(And the same for functions with finite support.) Here $C^{\infty}(\mathbb{R})$ is a space of smooth functions on $\mathbb{R}$ with a coarse topology (i.e., with a topology of a projective limit according to an increasing family of intervals), $C^{-\infty}(\mathbb{R})$ is a dual space to a space $C_{0}^{\infty}(\mathbb{R})$ of smooth functions with compact support with the topology of an inductive limit with respect to this family of interval (the space $C_{0}^{\infty}(\mathbb{R})$ is often denoted as $\mathscr{D}$, and the space $C^{-\infty}(\mathbb{R})$ as $\left.\mathscr{D}^{\prime}\right)$.

This pair of topologies on functions defines a pair of topologies on the space of IDS. Taking as a dual space in this category to the pair $V_{+} \subset V_{-}$the pair 
$V^{*} \subset V_{+}^{*}$, we get a formal justification of what was said above: a differential of a "smooth function" on $V_{+}$, i.e., a smooth function on $V_{-}$, corresponds in a described above way to a pseudodifferential symbol with smooth coefficients, and not with generalized coefficients as in the naïve theory.

In the same way we can consider the $\Psi D S$ with finite support (or with rapidly decreasing coefficients) by introduction of the pair $C_{c}^{\infty}(\mathbb{R}) \subset C_{c}^{-\infty}(\mathbb{R}) .{ }^{9}$ Such a play with topologies is a usual deal in the theory of infinite-dimensional manifolds. Several other examples will be given in what follows. We should note that any smooth function on the smaller set of $\Psi D S$ (i.e., on the set of $\Psi D S$ with smooth coefficients) extends uniquely to the bigger one (i.e., of $\Psi D S$ with generalized coefficients) by definition, however, only the smaller set carries an algebra structure. $^{10}$

Now we discuss the (much simpler) theory in the case of the group $\operatorname{GL}\left(C^{\infty}(\mathbb{R})\right)$. We should note however, that the only explanation we are going to give is the explanation of the choice of the dual space to the Lie algebra of this group. We cannot explain even such a simple question as what is the relation between the Lie algebra we consider and the Lie group we consider, or what is the topology on this Lie algebra. The only hope we have now is that the discussion below can justify the identification of the space we considered in Sect. 2.1 to be a dual space to the Lie group. However, if we could find some good set of smooth functions on $\mathrm{GL}\left(C^{\infty}(\mathbb{R})\right)$ in the same sense as below, then the hypothetical Hopf algebra structure on this vector space could justify all the algebraic discussion above. This is related to the fact that the language of Hopf algebras allows one to live quite comfortable without a reference to the Lie algebra, if we have a good description of functions on the group, and, in particular, of the dual space to the Lie algebra.

We begin the discussion with a note that formula (2.3) determines the $r$-matrix from the algebraic point of view. However, to be able to apply all the described above machinery we should verify that this is indeed an $r$-matrix, therefore we should show that this formula has something to do with $\mathfrak{g} \otimes \mathfrak{g}$. The problem is that this element is "outside" of any naturally defined notion of the tensor square for any naturally defined notion of the vector space $g l\left(C^{\infty}(\mathbb{R})\right)$.

In the finite dimensional case if we consider a vector subspace, then the dual space to the subspace is "smaller" than the dual space to the ambient space. The same is true for closed subspaces in the topological case. However, for a dense inclusion the dual space to the subspace is bigger than the dual space to the ambient space. On the other hand, the definition of the biggest possible tensor square is the space of bilinear functionals on the dual space. Therefore to get the given element into $\mathfrak{g} \otimes \mathfrak{g}$ we can take the smallest possible $\mathfrak{g}^{*}$, i.e., the biggest

\footnotetext{
9 This should be the choice if we considered the second Gelfand-Dickey structure in the context of integrable systems, since the usual local Hamiltonians are defined on this space

${ }^{10}$ Let us give here another example of a similar situation. In the theory of quantum groups one considers an algebra (or a Hopf algebra) of generalized functions on a group $G$ with support in $e \in G$. This space carries a topology of an inductive limit. However, if one considers the dual Hopf algebra, it is not the dual space of $\infty$-jets of functions at $e \in G$, which carries a topology of a projective limit, but the subspace spanned by the matrix coefficients in finite-dimensional representation, that carries a topology of an inductive limit. If the group $G$ is a linear algebraic group, the latter space coincides with polynomial functions on $G$
} 
possible $\mathfrak{g}$, and the biggest possible notion of the tensor square. However, this is not sufficient.

Therefore we apply the following trick: we take two different definitions of $\mathrm{gl}\left(C^{\infty}(\mathbb{R})\right)$ that result in two topological vector spaces with an intersection that is dense in both spaces. We consider the sum of these two vector spaces as $\mathfrak{g}$, more precise, we consider a linear functional on this sum to be an element of $g^{*}$. This sum does not carry a structure of a Lie algebra, however, below we propose some way of interpretation of this trick.

Denote these two Lie algebras as $\mathfrak{g}_{1}$ and $\mathfrak{g}_{2}$. Consider the corresponding Lie groups $G_{1}$ and $G_{2}$ and the group $G_{12}=G_{1} \cap G_{2}$. Now call a function on $G_{12}$ a smooth function if this function can be extended as a smooth function on both $G_{1}$ and $G_{2} \cdot{ }^{11}$ In this definition the tangent space to $G_{12}$ at $e$ is $\mathfrak{g}_{1} \cap \mathfrak{g}_{2}$, but the cotangent space (i.e., the space of values of differentials of functions) is $\mathfrak{g}_{1}^{*} \cap \mathfrak{g}_{2}^{*}=\left(\mathfrak{g}_{1}+\mathfrak{g}_{2}\right)^{*}$. Therefore if $r$ is a bilinear functional on $\mathfrak{g}_{1}^{*} \cap \mathfrak{g}_{2}^{*}$, we can compute the value of $r$ on any pair of differentials of functions. Therefore we can eventually define the Poisson bracket of (smooth!) functions on $G_{1} \cap G_{2}$. Now we proceed with the description of the spaces $\mathfrak{g}_{1}$ and $\mathfrak{g}_{2}$.

If the pair $C^{\infty}(\mathbb{R}) \subset C^{-\infty}(\mathbb{R})$ represents the space of functions on a line, then instead of a group of continuous operators in the space of functions on $\mathbb{R}$ we should use the set of continuous operators in this pair, i.e., the set of pairs of maps

$$
\begin{gathered}
T_{\infty}, T_{-\infty}, \quad T_{\infty}: C^{\infty}(\mathbb{R}) \rightarrow C^{\infty}(\mathbb{R}), \quad T_{-\infty}: C^{-\infty}(\mathbb{R}) \rightarrow C^{-\infty}(\mathbb{R}), \\
\left.T_{-\infty}\right|_{C^{\infty}(\mathbb{R})}=T_{\infty} .
\end{gathered}
$$

Hence the group we consider is $\operatorname{GL}\left(C^{\infty}(\mathbb{R})\right) \cap \operatorname{GL}\left(C^{-\infty}(\mathbb{R})\right)$, and the pair of group inclusions is

$$
\begin{aligned}
& G_{12}=\mathrm{GL}\left(C^{\infty}(\mathbb{R})\right) \cap \mathrm{GL}\left(C^{-\infty}(\mathbb{R})\right) \subset \mathrm{GL}\left(C^{\infty}(\mathbb{R})\right)=G_{1}, \\
& G_{12}=\mathrm{GL}\left(C^{\infty}(\mathbb{R})\right) \cap \mathrm{GL}\left(C^{-\infty}(\mathbb{R})\right) \subset \mathrm{GL}\left(C^{-\infty}(\mathbb{R})\right)=G_{2} .
\end{aligned}
$$

Correspondingly instead of the dual space to a Lie algebra of continuous operators in the space of functions we will take a dual to a pair of inclusions

$$
\begin{aligned}
& \mathfrak{g}_{12}=\mathfrak{g l}\left(C^{\infty}(\mathbb{R})\right) \cap \operatorname{gl}\left(C^{-\infty}(\mathbb{R})\right) \subset \mathfrak{g l}\left(C^{\infty}(\mathbb{R})\right)=\mathfrak{g}_{1}, \\
& \mathfrak{g}_{12}=\mathfrak{g l}\left(C^{\infty}(\mathbb{R})\right) \cap \operatorname{gl}\left(C^{-\infty}(\mathbb{R})\right) \subset \mathfrak{g l}\left(C^{-\infty}(\mathbb{R})\right)=\mathfrak{g}_{2},
\end{aligned}
$$

i.e., a pair of inclusions

$$
\begin{aligned}
& \left(\mathfrak{g l}\left(C^{\infty}(\mathbb{R})\right) \cap \operatorname{gl}\left(C^{-\infty}(\mathbb{R})\right)\right)^{*} \supset \mathfrak{g l}\left(C^{\infty}(\mathbb{R})\right)^{*}, \\
& \left(\mathfrak{g l}\left(C^{\infty}(\mathbb{R})\right) \cap \operatorname{gl}\left(C^{-\infty}(\mathbb{R})\right)\right)^{*} \supset \mathfrak{g l}\left(C^{-\infty}(\mathbb{R})\right)^{*} .
\end{aligned}
$$

We need all this machinery to find an appropriate definition of the space

$$
\Lambda^{2}(\mathfrak{g l}(\text { functions on } \mathbb{R})) \text {. }
$$

Without it we could define the formulae for the $r$-matrix we want to construct, but we could not motivate a choice of (a big) space this matrix lies in. This is a usual difficulty in functional analysis. It is difficult to define a notion of a tensor product

\footnotetext{
11 Of course, we need to know first what is a smooth function on, say, $G_{1}$. However, here we skip this discussion (until a better time)
} 
of a pair of spaces. It is possible in the case when one of these spaces is a nuclear space. However, in our case we want these spaces to be Lie algebras, and these two restrictions (being a Lie algebra and a nuclear space) seem to contradict in this particular case (at least we could not invent anything feasible).

So now we can define the space $\Lambda^{2}($ gl(functions on $\left.\mathbb{R})\right)$ to be the space of skewsymmetric continuous bilinear forms on the space

$$
\mathfrak{g l}\left(C^{\infty}(\mathbb{R})\right)^{*} \cap \operatorname{gl}\left(C^{-\infty}(\mathbb{R})\right)^{*} \subset\left(\mathfrak{g l}\left(C^{\infty}(\mathbb{R})\right) \cap \mathfrak{g l}\left(C^{-\infty}(\mathbb{R})\right)\right)^{*} .
$$

This space is sufficiently small for the formula (2.3) to define a continuous bilinear form on it. Indeed, as we explained in Sect. 2.1, the kernels of covectors from this set are smooth with compact support in one variable. We hope that this sketch can help in justification of our consideration of (2.3) as of an $r$-matrix.

\section{References}

1. Adler, M.: On a trace functional for formal pseudodifferential operators and the Hamiltonian structure of Korteweg-de Vries type equations. Global analysis (Berlin), Lecture Notes in Math., vol. 755, Proc. Biennial Sem. Canad. Math. Congr., Univ. Calgary, Calgary, Alta. 1978, Springer 1979 , pp. $1-16$.

2. Drinfeld, V.G.: Hamiltonian structures on Lie groups, Lie groups, Lie bialgebras and the geometric meaning of classical Yang-Baxter equations. Doklady Akademii Nauk SSSR 268, no. 2, 285-287 (1983) (Russian)

3. Drinfeld, V.G., Sokolov, V.V.: Lie algebras and equations of Korteweg-de Vries type, Current problems in mathematics (Moscow), Itogi Nauki i Tekhniki, vol. 24, Akad. Nauk SSSR, Vsesoyuz. Inst. Nauchn. i Tekhn. Inform., 1984, pp. 81-180 (Russian)

4. Feigin, B.L., Edward Frenkel: Semi-infinite Weil complex and the Virasoro algebra. Commun. Math. Phys. 137, 617-639 (1991)

5. Israel M. Gelfand, Leonid A. Dickey: On the second Hamiltonian structure for equations of Korteweg-de Vries type. Preprint. The Institute for Applied Mathematics, Moscow, USSR 1978

6. Boris Khesin, Ilya Zakharevich: The Gelfand-Dickey structure and an extension of the Lie algebra of pseudodifferential symbols. In preparation

7. Jiang-Hua Lu, Alan Weinstein,: Poisson Lie groups, dressing transformations, and Bruhat decompositions. J. Differ. Geom. 31, 501-526 (1990)

8. Radul, A.O.: Nontrivial central extensions of Lie algebras of differential operators in two and higher dimensions. Phys. Lett. B 265, 86-91 (1991)

9. Semenov-Tyan-Shanskii, M.A.: What a classical $r$-matrix is. Akademiya Nauk SSSR. Funktsionalnyi Analiz i ego Prilozheniya 17, 17-33 (1983) (Russian)

10. Semenov-Tyan-Shanskii, M.A.: Dressing transformations and Poisson group actions. Kyoto University. Research Institute for Mathematical Sciences. Publications 21, 1237-1260 (1985) 
